\title{
Magnetic Compensation of Magnetic Noises Related to Aircraft's Maneuvers in Airborne Survey
}

\author{
R. W. Groom, PetRos EiKon, Concord, Ontario, Canada \\ Ruizhong Jia, PetRos EiKon, Concord, Ontario, Canada \\ Bob Lo, BHL Earth Sciences, Thornhill, Ontario, Canada
}

\begin{abstract}
For a variety of applications, magnetic data is collected from airborne platforms. Normally, this data is collected with sensors that measure the total field or amplitude of the magnetic vector data. New generations of optically pumped sensors are extremely sensitive with their sensitivity quoted often in picoteslas. At present, some new instrumentation is also attempting to measure high accuracy vector data. Despite the accuracy of modern sensors and data acquisition systems, the noise of the flying platform is still one of the limiting factors in obtaining highly accurate data.

The aircraft or helicopter itself emanates magnetic signals. These signals are due to a number of factors including induced fields due to magnetically susceptible materials and permanent magnetic materials on the platforms as well as both induced electromagnetic signals and electromagnetic signals generated either by electrical systems or moving parts such as rotors.

This subject of this paper are problems and techniques related to removing the effects of the moving platform as well as attempts to study the subject with the use of simulated data.
\end{abstract}

\section{Introduction}

In 1961, Leliak (1961) published a paper outlining a mathematical technique for estimating and removing the effects of an aircraft from magnetic data collected from a served total field sensor. The basis of this technique is a system of linear equations which attempts to represent the permanent, induced and EM effects due to an aircraft as a function of the attitude. Four flightpaths are normally flown at high altitude in a box wide sides parallel, and perpendicular paths to the proposed survey azimuths with a series of pitch, roll and yaw movements. The data from these flight paths are then used to determine up to 18 coefficients relating to the motions and the linear equation system is then usually solved with a least-squares method. The resulting coefficients are then used to remove the aircraft's magnetic effects from survey data flown at lower altitude. The attitude of the plane is usually determined from vector fluxgate data from sensors also mounted on the aircraft but attempts are being made to determine attitude from multiple GPS sensors.

Later papers by Leach $(1979 a, 1979 b)$ discussed the variety of factors affecting the accuracy of the so-called "compensation" of the aircraft including the orthogonality of the fluxgate sensors, the effects of regional gradients as well as techniques for solving the equations for the coefficients. Leach studied the sensitivity of the coefficients as a function of band pass filtering and introduced 3 gradient terms into the 18-term model to establish a complete interference model involving 21 terms. To improve the computation of the coefficients, he experimented with various techniques to solve 16 and 18-term linear equation system, such as, stepwise regression analysis, rank deficient singular value decomposition, and ridge regression analysis. It has been demonstrated that application of ridge regression analysis permits useful and stable compensation results.

As part of the process of determining compensation coefficients from the flight box data, it is generally considered necessary to highpass the data to remove the lower frequency information from the 
data to provide more accurate and stable solutions. We have implemented the Gaussian high pass filter which enables us to better control the filtration process utilized in the coefficients computation. It is concluded that good compensation coefficients can be generated by applying filtering to total field and fluxgate data rather than to the linear operator matrices as is traditionally done. We also concluded that good interference coefficients can be generated with all the box lines. Our capability of building synthetic model is invaluable to reveal the relationship between the mathematical theory and physical factors and thus provides useful guidelines for our work We have been studying the suitability of the basic equations through the use of synthetic data, the variation of solutions with different mathematical techniques for solving the equations as well as the use of GPS orientation information. In particular we have demonstrated that ridge regression analysis and truncated singular value decomposition are very effective techniques to improve the predicative power of the 16-term and 18-term interference models, especially in the case that there exist multicolinearities in the interference models.

\section{Compensation Coefficients Computation}

\section{Mathematical model for Aircraft Interference}

The following 18 term aircraft interference model was presented by Leliak (P. Leliak, 1961):

$$
\begin{aligned}
H_{T}=c_{1} & \cos X+c_{2} \cos Y+c_{3} \cos Z+ \\
& +H_{e}\left\{c_{4} \cos ^{2} X+c_{5} \cos X \cos Y+c_{6} \cos X \cos Z+\right. \\
& \left.+c_{7} \cos ^{2} Y+c_{8} \cos Y \cos Z+c_{9} \cos ^{2} Z\right\}+ \\
& +H_{e}\left\{c_{10} \cos X(\cos X)^{\prime}+c_{11} \cos X(\cos Y)^{\prime}+c_{12} \cos X(\cos Z)^{\prime}+\right. \\
& +c_{13} \cos Y(\cos X)^{\prime}+c_{14} \cos Y(\cos Y)^{\prime}+c_{15} \cos Y(\cos Z)^{\prime}+ \\
& \left.+c_{16} \cos Z(\cos X)^{\prime}+c_{17} \cos Z(\cos Y)^{\prime}+c_{18} \cos Z(\cos Z)^{\prime}\right\}=\sum_{i=1}^{18} c_{i} a_{i},
\end{aligned}
$$

where $H_{e}$ is the earth's magnetic field, $(\cos X, \cos Y, \cos Z)$ is the directional cosines of the earth's field vector with the transverse, longitudinal and vertical axes of aircraft, ()$^{\prime}$ is the differentiation operator with respect to fiducial, $H_{T}$ is the total intensity of the interference field, $c_{i}, 1 \leq i \leq 18$, are the desired coefficients. Once the coefficients are obtained we have an explicit model for predicting aircraft interference; interference effects are eliminated by subtracting the calculated $H_{T}$ from the magnetometer signal.

Note that the first 3 term represent the permanent source interference field, followed by 6 terms standing for the induced source interference field, and the last 9 terms express the eddy-current interference. In other words, $c_{i}, 1 \leq i \leq 3$, are for the permanent source interference field, and $c_{i}$, $4 \leq i \leq 10$, are for the induced source interference field, and $c_{i}, 10 \leq i \leq 18$, are for the eddy-current interference. Substituting the following two identities (the second one is derived from the first one)

$$
\begin{aligned}
& \cos ^{2} X+\cos ^{2} Y+\cos ^{2} Z=1, \\
& \cos X(\cos X)^{\prime}+\cos Y(\cos Y)^{\prime}+\cos Z(\cos Z)^{\prime}=0
\end{aligned}
$$

into Equation (1) eliminates two terms and thus yields the 16-term model.

Consider now $N(N>18)$ samples of total field data and fluxgate data to be measured with aircraft maneuvering. Then the linear equation system can be expressed in terms of matrix notation

$$
A C=Y \text {, }
$$


where $A=\left(\begin{array}{cccc}a_{1}(1) & a_{2}(1) & \cdots & a_{18}(1) \\ a_{1}(2) & a_{2}(2) & \cdots & a_{18}(2) \\ \vdots & \vdots & & \vdots \\ a_{1}(N) & a_{2}(N) & \cdots & a_{18}(N)\end{array}\right), \quad C=\left(\begin{array}{c}c_{1} \\ c_{2} \\ \vdots \\ c_{18}\end{array}\right), \quad Y=\left(\begin{array}{c}H_{T}(1) \\ H_{T}(2) \\ \vdots \\ H_{T}(N)\end{array}\right)$.

\section{Filtering and Coefficients Computation}

In real airborne survey, there are many background noises of various types affecting the compensation process such as local gradient of total field, geologic noise, and micropulsation. The data has to be filtered in order to restrict the frequency range of the data, for the compensation process, to a band of frequencies centered around the primary frequency modes for the aircraft maneuvers. As a result, the signal-to-noise ratio is greatly enhanced for the aircraft maneuver interference that is generated in the magnetic signal. Usually the filtration process involves either high-pass or band-pass filtering.

To achieve this, Leach (B. W. Leach, 1979a, 1979b) suggested that the sampled data quantities, that is, each column of $A$ and the column vector $Y$ in (2), have identical filtering. In other words, filtration is applied to the matrices $A$ and $Y$ rather than to measured total field and fluxgate data. Based on our experiments with synthetic model as well as real compensation boxes. it is concluded that a good set of interference coefficients in a predictive model can be produced by first applying a Gaussian highpass filter directly to both the data and the fluxgate data and then adding a DC value (average value) to the filtered data. It is evident that adding DC values is essential as they specify the aircraft's orientation including the heading direction along which a particular flight path is flown.

With our real case study, we utilized the compensation box flown with a Piper Navajo by Terraquest. The earth's field in the survey region is inclined at 75 degrees to the horizontal and oriented 5 degrees to the north and has strength of $55500 \mathrm{nT}$. The flight altitude is 9500 feet. The four flight paths of the box are illustrated in Figure 1. The red flight line is from south to north, the blue from west to east, the green from north to south, and the brown from east to west. By retrieving data from each line in the box, coefficients were generated which were then applied to compensate the total field from each respective line. We call this compensation procedure line-to-line compensation. Some other ways of retrieving data for compensation will be discussed later. Figure 2 displays the line-to-line compensation results with two different filtration procedures mentioned above for portion of the red flight path. Before the compensation the peak-to-peak interference noise due to aircraft's maneuvers in the selected portion is about $9 \mathrm{nT}$ and it is reduced to less than $1 \mathrm{nT}$ by either methods. Furthermore, the absolute point-to-point difference between the two compensated results is within $0.5 \mathrm{nT}$.

We have developed the ability to generate a synthetic model of the aircraft's magnetic effects. Here, we simulated the sources of the permanent magnetic field in the aircraft with two magnetic dipoles, one on each wing. The sources of the induced magnetic field of the aircraft were simulated with 2 thin rectangular prisms representing the two wings and another long box type prism to represent the fuselage of the aircraft. We also simulated the eddy-current magnetic fields caused by the aircraft's conductive structures moving in the earth's magnetic field by calculating the time variation of the coupling of these prism structures with the earth's field. Here, the synthetic compensation box consists of 4 lines, each having a length of $14 \mathrm{~km}$, and are flown along EW and NS directions. The flight altitude of the box was set 9500 feet and the data was collected every $0.05 \mathrm{~s}$ or the sampling rate is $20 \mathrm{~Hz}$. The maneuvers of the aircraft were simulated with pitch $\left( \pm 5^{\circ}\right)$, roll $\left( \pm 5^{\circ}\right)$ and yaw $\left( \pm 10^{\circ}\right)$. The earth field is set to be the same as the field survey mentioned above (just NE of Toronto, Canada). The 4 box lines are illustrated in Figure 3 as red, blue, green and brown lines. A survey line (pink line) was simulated at 
altitude of 650 feet with pitch $\left( \pm 5^{\circ}\right)$, roll $\left( \pm 5^{\circ}\right)$ and yaw $\left( \pm 10^{\circ}\right)$. A large local magnetic anomaly was inserted in the eastern part of the survey region to simulate a regional gradient in the average earth's field.

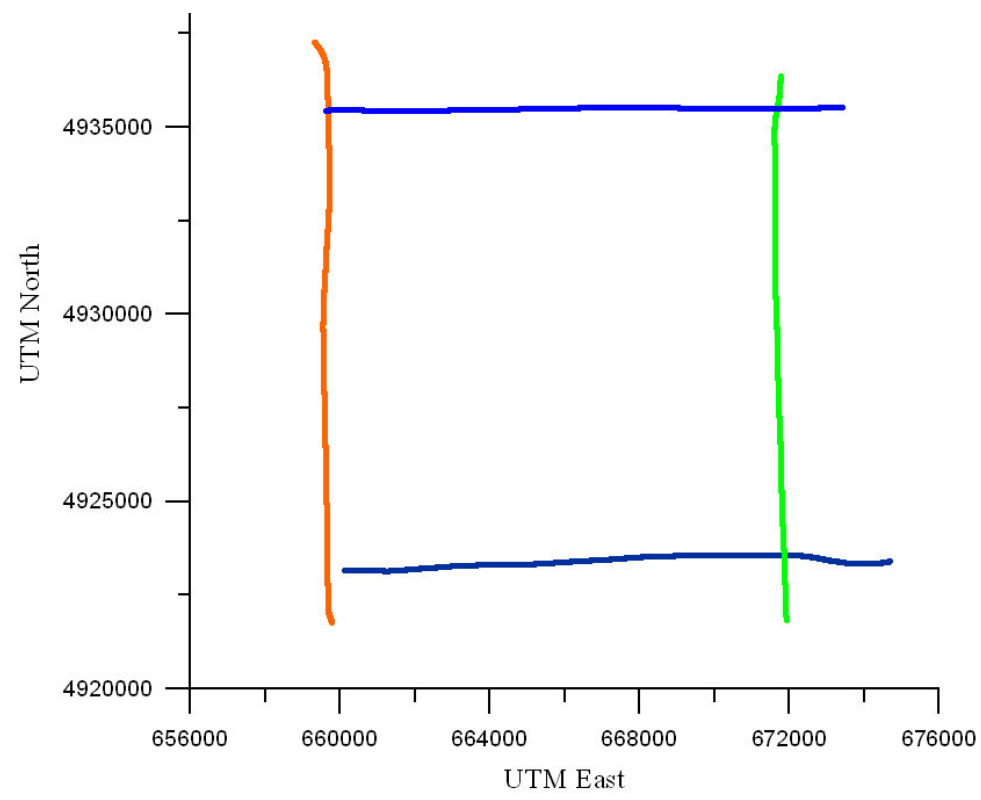

Figure 1: Compensation box

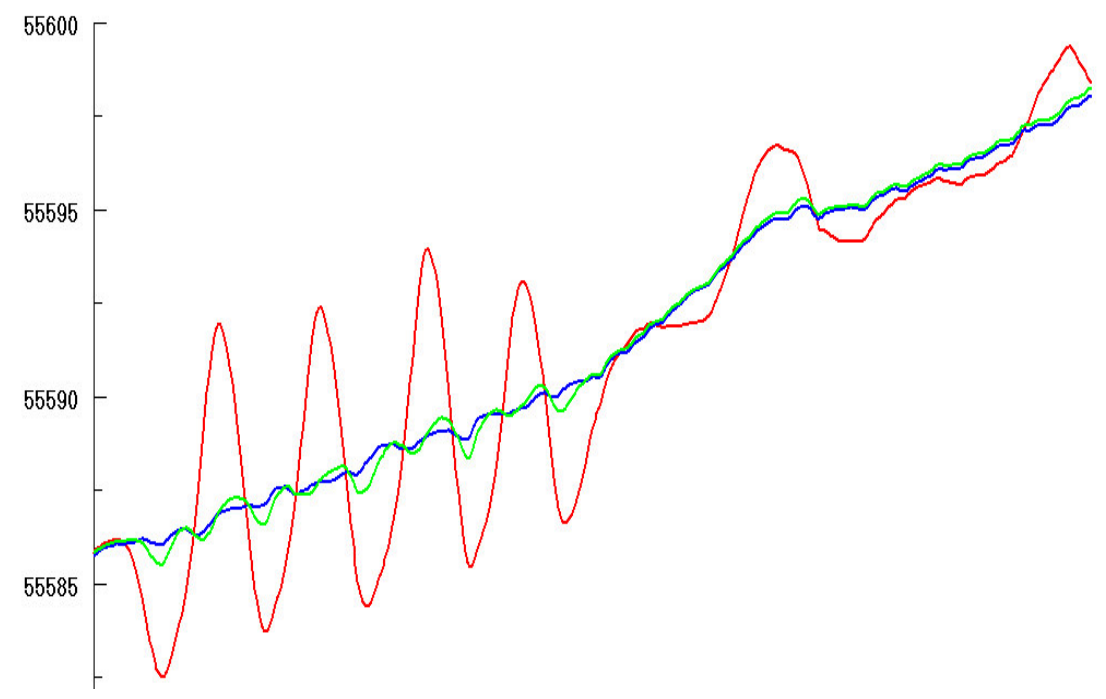

Figure 2: Real survey compensation results

Red curve: measured total field (nT)

Green curve: results by filtering data

Blue curve: results by filtering operator 


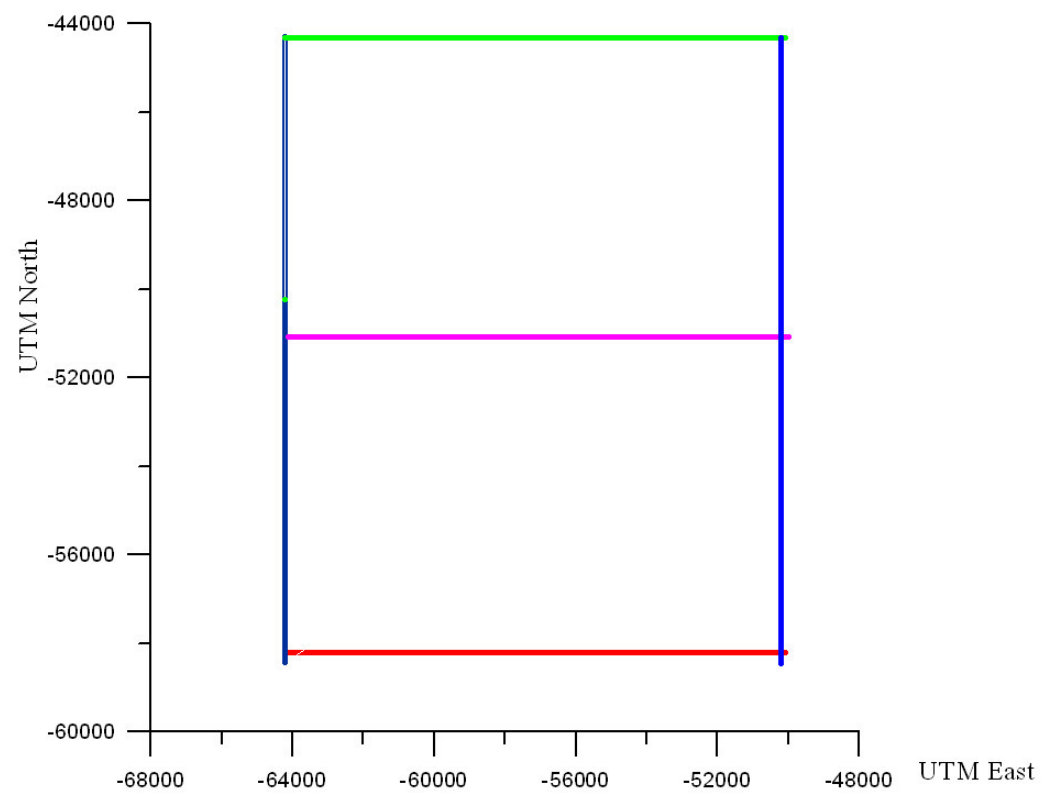

Figure 3: Synthetic compensation box and survey Line

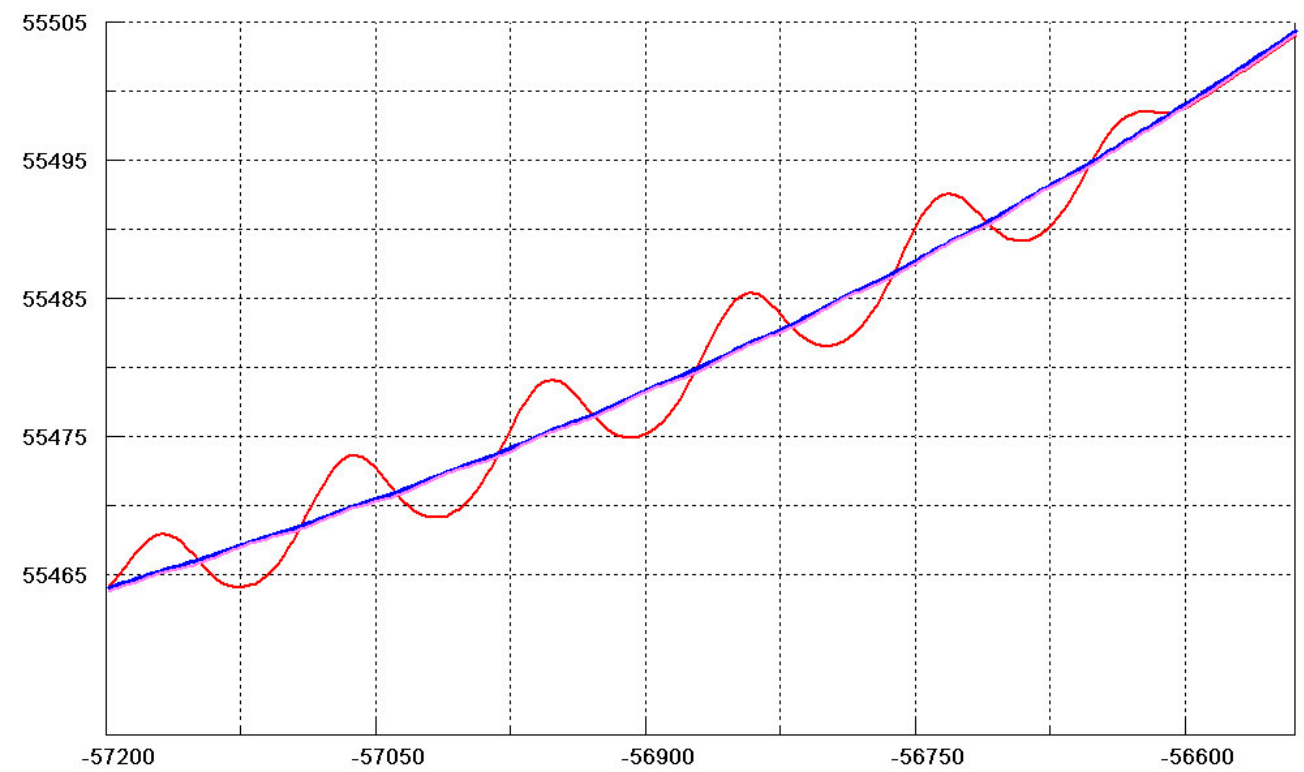

Figure 4: Compensation results with different filtration for synthetic model Red curve: measured total field (nT)

Blue curve: compensated results by filtering the total field and fluxgate data Pink curve: compensated results by filtering the linear operator matrix

We compensated the synthetic total field from the survey line (pink) using the coefficients derived from the "box" data along the southern EW line (red) which has the same heading direction as the survey line. For a selected portion of the survey line, Figure 4 displays the compensation results 
generated with two sets of coefficients; one set was produced by applying a Gaussian high pass filter to the total field and fluxgate data, and the other set was obtained by filtering the linear operator matrices. The compensation results generated with different filtration procedures are actually identical for this synthetic case.

Our results have shown we may generate reasonably good compensation coefficients by applying filtering to total field and fluxgate data rather than to the linear operator matrices. This enables us to better control the compensation process via data acquisition.

\section{Spectrum versus Accuracy}

To demonstrate the effect of filtering, we consider only the $5^{\text {th }}$ term in (Eqn 1), that is, $H_{e} \cos X \cos Y$. This term varies from point to point and is both a function of the aircraft attitude (i.e. fluxgate data) and the total field data. In Figure 5, the $5^{\text {th }}$ term generated from the real data is shown with a red line before filtration. The blue is the same term but after high pass filtering. In this case, the filter used is a Gaussian high pass filter with $0.2 \mathrm{~Hz}$ as the cut-off frequency. The signal-to-noise ratio was greatly enhanced for the aircraft maneuver interference after filtering which can be seen from spectrum plots.

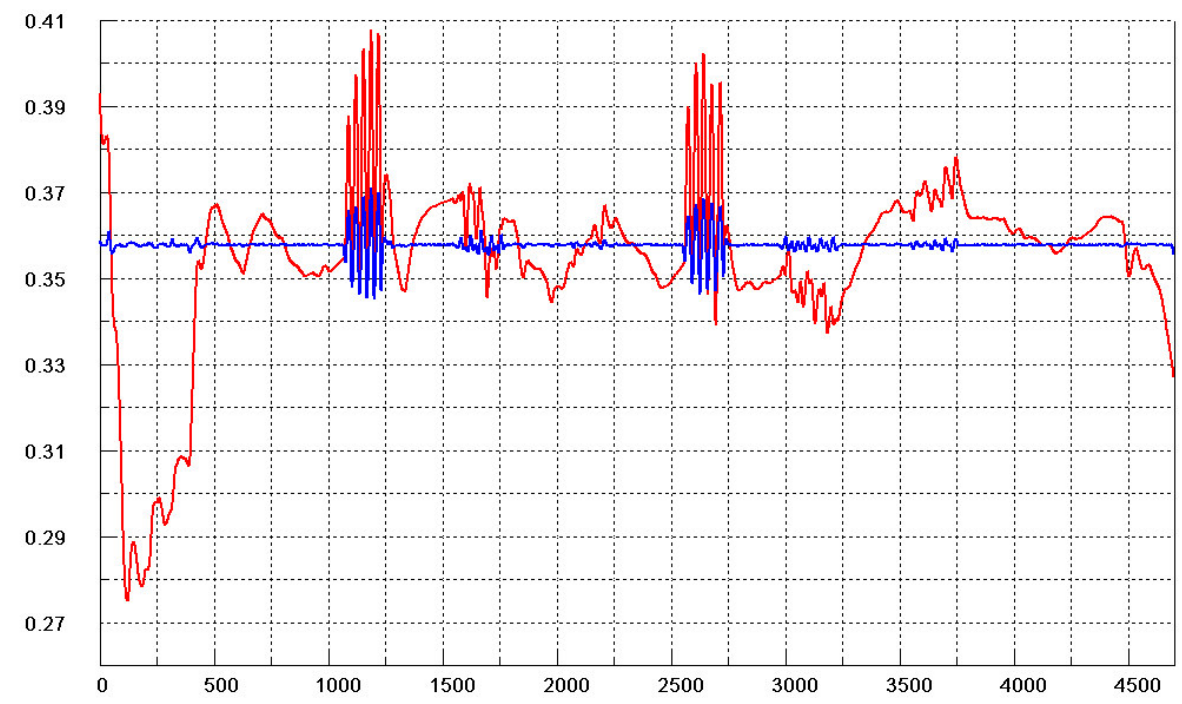

Figure 5: Fifth term in Equation (1) before and after filtration Red curve: $5^{\text {th }}$ term before filtration Blue curve: $5^{\text {th }}$ term after filtration

\section{Coefficients from All Box Lines}

In practice, once a compensation box is flown, a set of coefficients will be calculated with the data from each individual line of the box. To compensate the total field data of a particular survey line, which is at lower altitude, the set of coefficients of a box line, which has the closest heading as the survey line, is selected. We experimented with producing a single set of coefficients using the data of all four box lines and applying it to a survey line. Our results with both the synthetic model and real compensation box have shown that effective compensation can be achieved this way. 
We compared the line-to-line compensation results versus compensation by all the 4 box lines in Figure 6. The exactly the same portion of the red line of the real compensation box as that displayed in Figure 1 was used.

It is shown that the maximum absolute point-to-point difference between the two compensation results is within $0.5 \mathrm{nT}$. We also did testing with our synthetic model and there was little difference between the line-to-line compensation results and the results with all the 4 box lines. This method provides very important and practical implication, especially for the real time compensation.

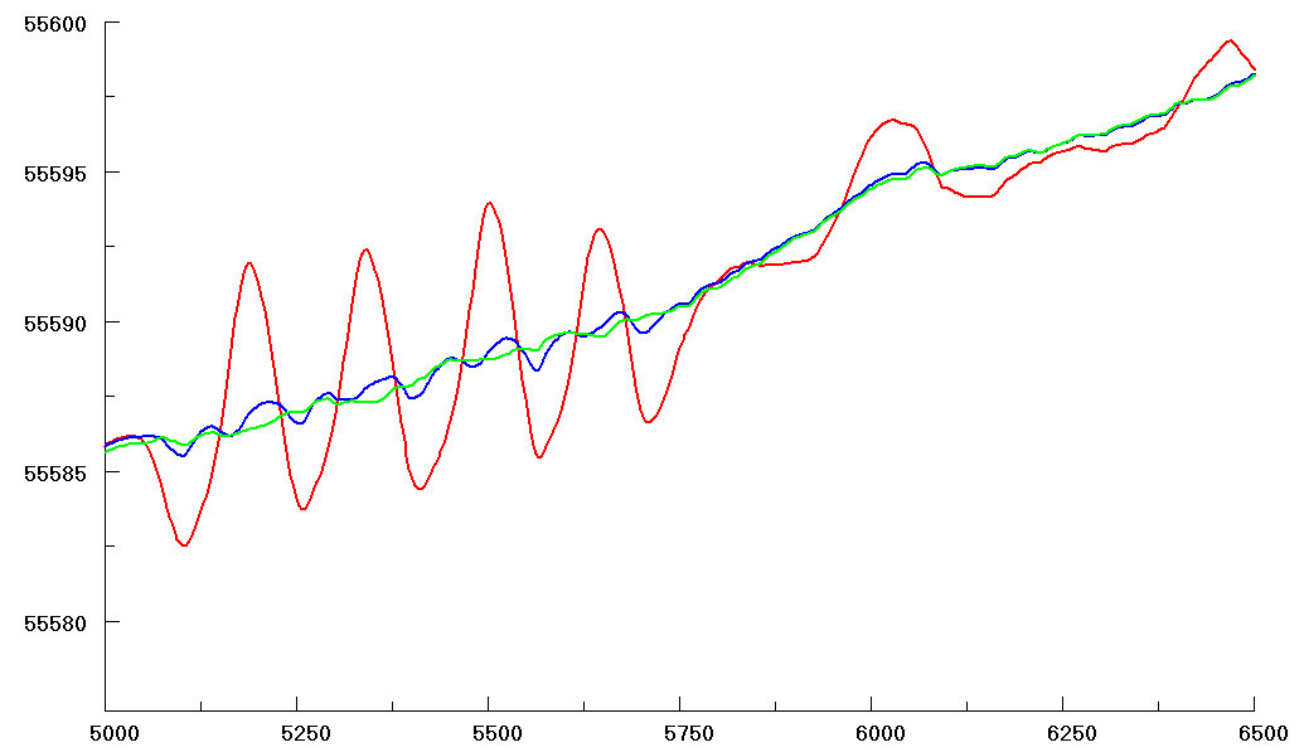

Figure 6: Compensation results with all box lines and single line

Red curve: measured total field (nT)

Blue: line-to-line compensation results

Green: compensation results with all four box lines

\section{Conclusion}

We implemented the Gaussian high pass filter which enables us to better control the filtration process utilized in the coefficients computation. It is concluded that good compensation coefficients can be generated by applying filtering to total field and fluxgate data rather than to the linear operator matrices. We also concluded that good interference coefficients can be generated with all the box lines.

Our capability of building synthetic model is invaluable to reveal the relationship between the mathematical theory and physical factors and thus provides useful guidelines for our work We have demonstrated that ridge regression analysis and truncated singular value decomposition are very effective techniques to improve the predicative power of the 16-term and 18-term interference models, particularly in the case that there exist multicolinearities in the interference models. The variation of solutions with different mathematical techniques for solving the equations as well as the use of GPS orientation information have been investigated and will be presented. 


\section{Acknowledgements}

We wish to thank the Ontario government for partially funding the project under the OMET project. To Terraquest Ltd for their assistance in collection of the data and to Barrie Leach and Nelson Bradley (NRC-Canada) for their helpful discussions and suggestions.

\section{References}

[1] P. Leliak, Identification and Evaluation of Magnetic Field Sources of Magnetic Airborne Detector MAD Equipped Aircraft, IRA Transactions on Airspace and Navigational Electronics, Volume 8, P.95105, September 1961.

[2] B. W. Leach, Automatic Aeromagnetic Compensation, National Research Council of Canada, National Aeronautical laboratory LTR-FR69, March 1979a.

[3] B. W. Leach, Aeromagnetic compensation as a linear regression problem, Information linkage between applied mathematics and industry II: Academic Press Inc., 1979b. 